$1^{\text {thi }}$

\title{
Initial Evidence of Lunar Mantle Composition Detected by Chang'e-4
}

\author{
By SONG Jianlan (Staff Reporter)
}

\author{
It marks the first step on the far side of the Moon; it \\ would never be resigned to playing second fiddle.
}

$\mathrm{P}$ revious research suggested that the lunar crust as well as the mantle could both have formed at the infantile stage of the Moon's evolution. At this stage, major crashes and the accretion process generated a great amount of energy that melted down the minerals and gave birth to a magma ocean. When this ocean cooled down, lighter substances, represented by the calcium-rich plagioclase, crystallized and rose to shape the crust, while the heavier ones, like the femic minerals including olivine and low-calcium pyroxene, sank down to form the mantle.

Curiously, the lunar samples returned by the Apollo and Luna missions did not give any direct information about the substantial composition of the mantle, leaving the above-mentioned inference unsupported for a long time. Still, the detection of such evidence is not impossible - under certain conditions the mantle substance could emerge to the surface and be picked up and recorded by man-made devices. Large craters left by big clashes on the Moon, scientists conceive, might provide some clues, as the great impact from the clashes could have penetrated and ripped open the crust, exposing the mantle partially to the surface.

The mission Chang'e-t under China's Lunar

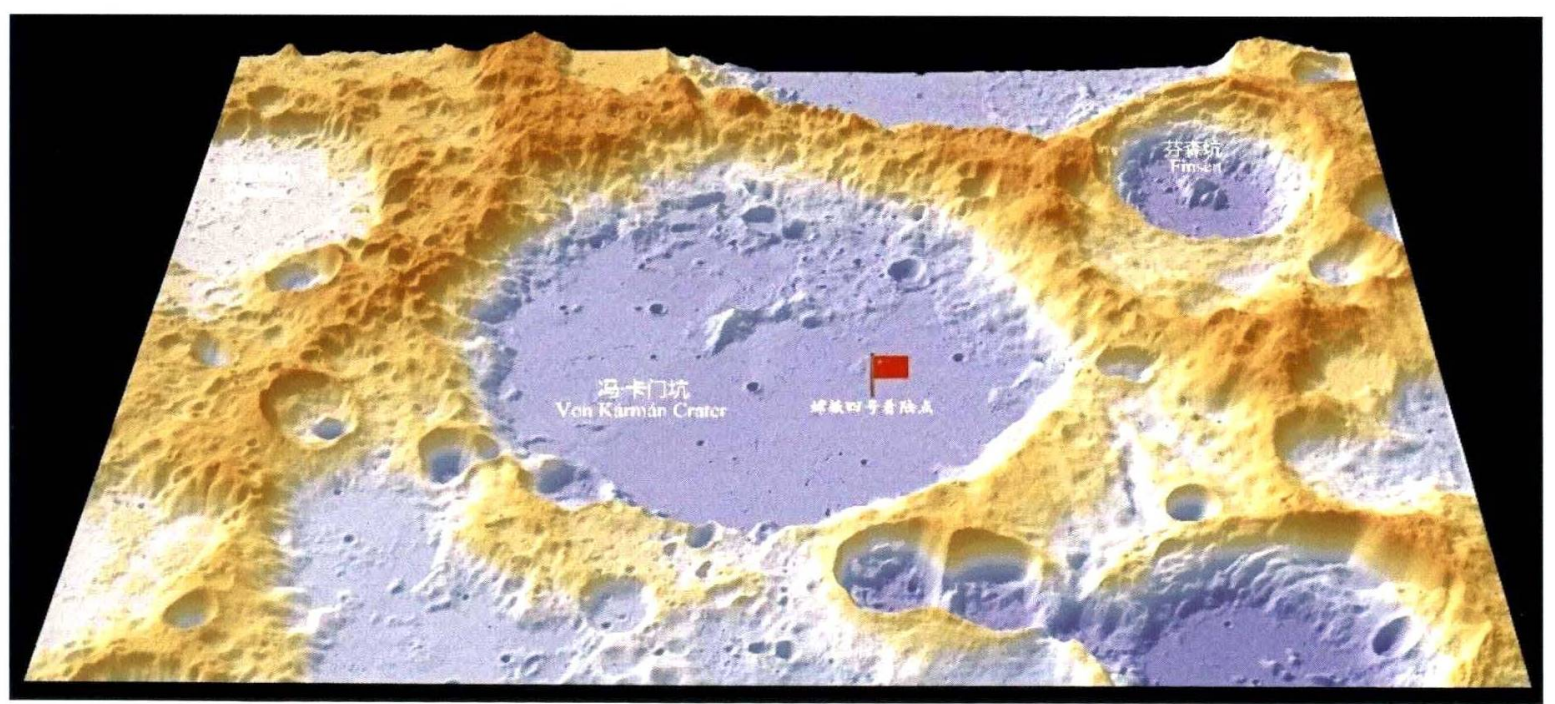

On January 9, 2019, Chang'e-4, a mission under China's Lunar Exploration Program, touched down on the eastern floor of the Von Kármán crater of the Moon's far side, and sent hours later the rover Yutu-2 to carry out an in-situ exploration of the local environment, the first ever made by a man-made spacecraft. It successfully detected initial evidence for the composition of the lunar mantle. Shown here is the landing site with possible mantle materials built up. (Credit: CLEP/GRAS/NAOC) 
Exploration Program (CLEP). helped to fill this gap with its in-situ investigation - the first ever by human beings - in the South Pole-Aitken basin (SPA) on the far side of the Moon. unveiling the first clips of this immersed world. This work, published in May 2019 in Nature, ranks first in the annual science advances of China for 2019.

Measuring $2.500 \mathrm{~km}$ in diameter. SPA ranks the oldest and the largest impact basin on the far side of the Moon. Very likely, the great impact that has shaped it has also penetrated the lunar crust and caused some buildup of materials from the mantle. However, existing remote sensing data from lunar orbiters gives no sign of widespread olivine, despite the signals indicating abundant femic minerals. Therefore, whether or not such substance has originated from the mantle was left open.

On January 9. 2019. Chang'e-t successfully landed at the Von Kármán crater of this area, and sent the rover Yutu-2 to probe this area. Based on the data obtained by the visible and near-infrared imaging spectrometer (VNIS) aboard Yutu-2, a team led by Prof. LI Chunlai from the National Astronomical Observatories (NAOC), CIS reported. together with their collaborators, the initial evidence for the existence of orthopyroxene and olivine in this area. indicating possible mantle origin of the accumulative materials near the landing site. According to their further background analysis, these mantle materials could have been projected from the $7.2 \mathrm{~km}$-diameter Finsen crater near to the landing site of Change-t.

Aside from revealing the possible composition of lunar mantle substance, this work has also improved the understanding of the formation and evolution of the lunar interior, and offered new constraints for the research into the early magma ocean.

Yutu-2, which is still roving on the Moon, will continue fact-finding mission at the bottom of the Von Kármán crater, aimed at understanding their geological background, origin and composition, laying a foundation for future sample-return missions of the CLEP. The beginning of 2020 has seen another discovery published concerning the lunar subsurface structure. based on Yutu2 's first two lunar days' investigation. The results reveal the shallow subsurface structure of the lunar strata of this area. presenting the first high-resolution image of a lunar ejecta sequence ever produced and the first direct measurement of its thickness and internal architecture.

For more about CLEP, please turn to page 27 in this issue.

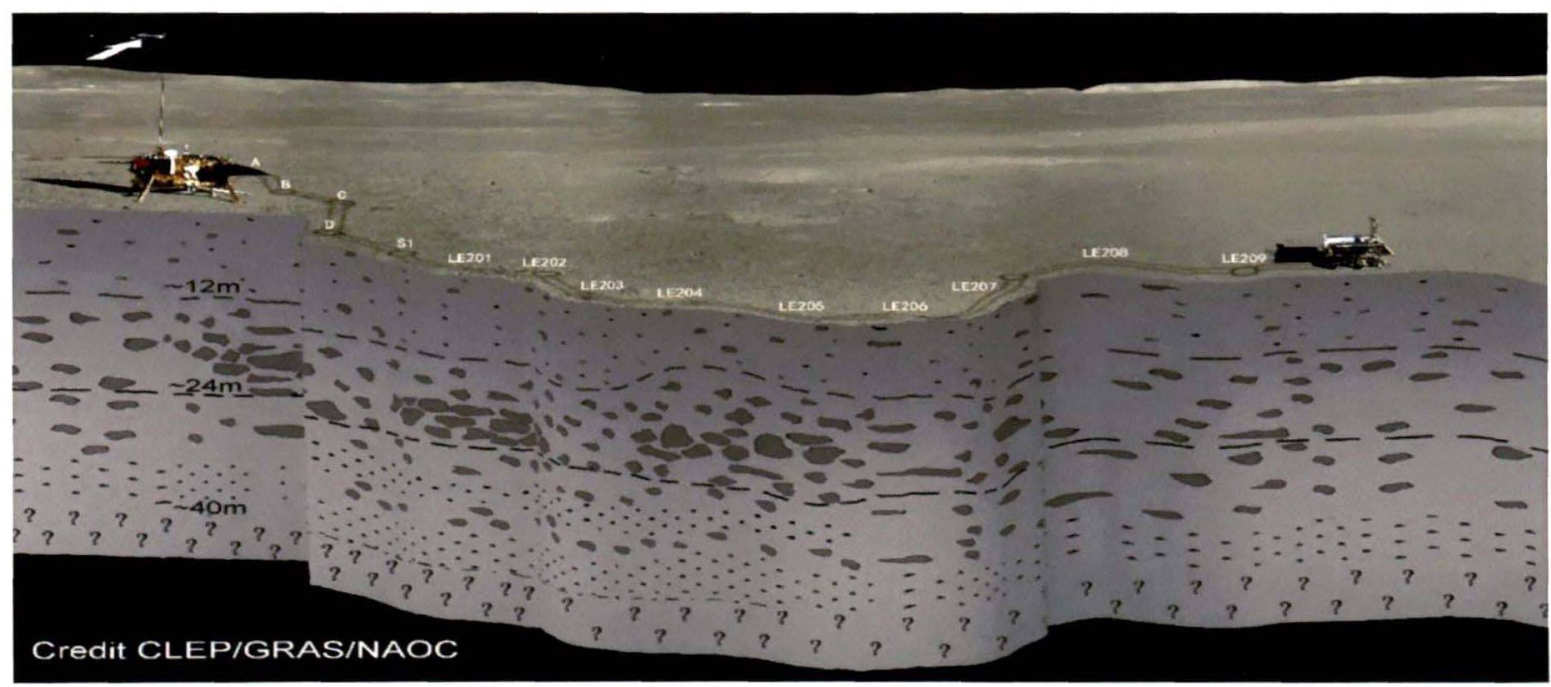

A joint team led by NAOC published February 27 in Science Advances the ensuing discovery by Yutu-2, revealing the shallow subsurface structure of the lunar strata near the landing site. (Credit: CLEP/GRAS/NAOC)

\section{Reference}

Chunlai Li*, Dawei Liu, Bin Liu, Xin Ren*, Jianjun Liu*, Zhiping He, Wei Zuo, Xingguo Zeng, Rui Xu, Xu Tan, Xiaoxia Zhang, Wangli Chen, Rong Shu, Weibin Wen, Yan Su, Hongbo Zhang \& Ziyuan Ouyang. (2019) Chang'E-4 initial spectroscopic identification of lunar far-side mantlederived materials. Nature, Vol. 569, 378-382.

Chunlai Li, Yan Su*, Elena Pettinelli*, Shuguo Xing*, Chunyu Ding, Jianjun Liu, Xin Ren, Sebastian E. Lauro, Francesco Soldovieri, Xingguo Zeng, Xingye Gao, Wangli Chen, Shun Dai, Dawei Liu, Guangliang Zhang, Wei Zuo, Weibin Wen, Zhoubin Zhang, Xiaoxia Zhang and Hongbo Zhang. (2020) Science Advances 6(9), eaay6898 (DOI: 10.1126/sciadv.aay6898) 


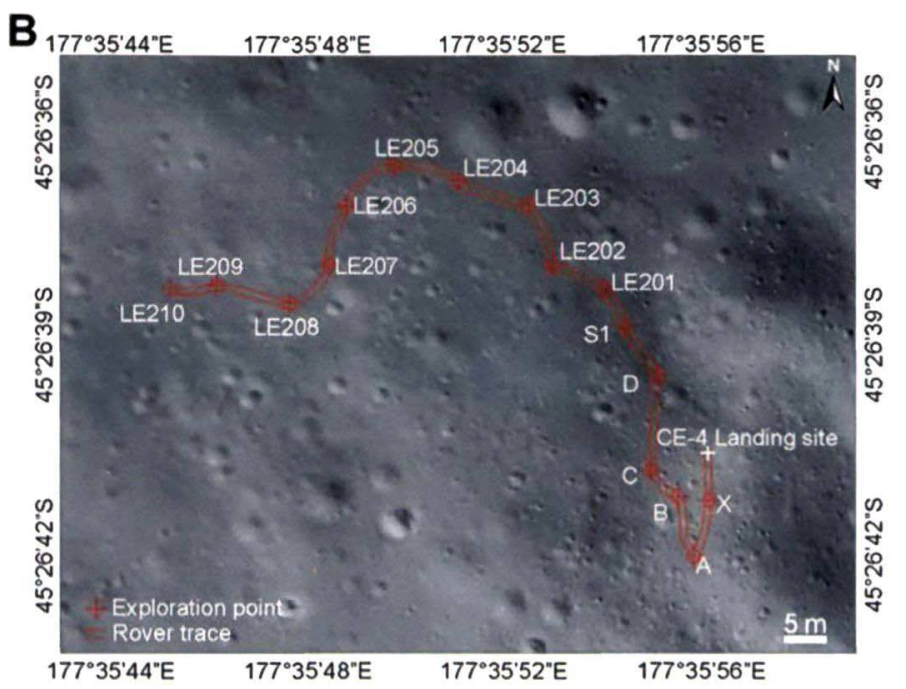

A series of exciting discoveries have been made concerning the chemical composition of the lunar mantle and the shallow subsurface structure of a lunar ejecta sequence, thanks to the data obtained during the in-situ observations performed by Yutu-2, the rover of Chang'e-4 mission under China's Lunar Exploration Program. The spacecraft touched down on the eastern floor of Von Kármán crater $\left(44.45^{\circ} \mathrm{S}, 176.3^{\circ} \mathrm{E}\right)$ in January 2019 , marking the first-ever soft landing by a manmade spacecraft on the far side of the Moon. The red lines, extending from point $A$ on the low right to point LE210 on the left, indicate the tracks of Yutu-2's left and right wheels during the first two lunar days. See page 4 and 8 for more. (Credit: CLEP/GRAS/NAOC) 